\title{
Revisión sistemática de intervenciones digitales para la reducción del consumo de cannabis
}

\author{
Cuauhtémoc Muñoz Ruiz' \\ ICentros de Integración Juvenil, México
}

\section{RESUMEN}

Introducción: la cannabis es una de las drogas que más se consume en el mundo y por la que hay mayor demanda de tratamiento. Los usuarios han identificado dificultades para acceder a tratamientos tradicionales, entre ellas el transporte, los costos y el estigma social. En el ámbito de la salud existen intervenciones digitales que usan la tecnología para facilitar la implementación de tratamientos; algunas se enfocan en la reducción del consumo de drogas, específicamente de cannabis; éstas podrían contribuir a solventar la demanda de tratamiento y a reducir las dificultades reportadas por los usuarios. Objetivo: actualizar el conocimiento acerca de la efectividad de las intervenciones digitales para reducir el consumo de cannabis, a través de una revisión sistemática. Método: en octubre de 2017 se emprendió una búsqueda sistemática en las siguientes fuentes: Cochrane Central Register of Controlled Trials, PsycINFO y PubMed; el intervalo de búsqueda fue 2010-2017. Resultados: fueron identificados 1022 registros, de los cuales se eligieron 26 ensayos clínicos para su análisis en la presente revisión. Discusión: la mitad de los ensayos analizados reportaron reducción en el consumo de cannabis asociado con una intervención digital. Hay algunas características frecuentes en las intervenciones; por ejemplo, la mayoría usa Internet para su entrega, se emplea la entrevista motivacional como enfoque teórico y gran parte de ellas se realizan en una única sesión. Ninguna de estos atributos u otro fue constante en las intervenciones efectivas. Conclusiones: existen intervenciones digitales efectivas para la reducción del consumo de cannabis. Se requiere mayor investigación para determinar las características que comprueban su efectividad, especialmente las que son propias de su condición digital.

Palabras clave: revisión sistemática, cannabis, uso de drogas, Internet, digital, intervención.

\begin{abstract}
ABCTRACT
Introduction: cannabis is one of the most popular drugs worldwide and request for it's treatment is high. Misusers have reported different obstacles to have access to typical treatments, transport, cost, social stigma, among others. In the public health field there are technological-approach interventions which are relatively easy to implement. Some of these interventions are focus on the reduction of drug misuse, specifically marijuana and it is believed that can reduce the issues reported by their users. Objective: to update the knowledge regarding to the effectiveness of technological-approach interventions which reduce marijuana misuse through a systematic review. Method: an specific search was started on October 2017 in the following databases: Cochrane Central Register of Controlled Trials, PsycINFO and PubMed. The searching interval was 2010-2017. Results: a total of 1022 papers were identified, from them 26 randomised clinical trials were selected for a posterior analysis in the present work. Discussion: a half of the analyzed assays report effectiveness of the technological-approach interventions in reducing cannabis use. Some characteristics among them are: Internet is the main way for their use and the motivational interview is the theoretical approach, and also many of them are one session only, although any of these was a constant in the effective interventions. Conclusions: there are effective technological-approach interventions to reduce marijuana misuse. However, more research is needed to identify the specific characteristics through which these interventions reach their effectiveness, specially related to the particularities of their digital/technological condition.
\end{abstract}

Key words: systematic review, cannabis, substance-related disorders, Internet, computer, intervention.

\footnotetext{
Autor de correspondencia:

Cuauhtémoc Muñoz Ruiz. Centros de Integración Juvenil. Zempoala núm. 77, col. Narvarte Oriente, del. Benito Juárez, C.P. 03020, Ciudad de México. Correo electrónico: cuauhtemoc.ruiz@cij.gob.mx, cuauhtemocmunozruiz@gmail.com

Recibido: 20 de marzo de 2018

Aceptado: 4 de mayo de 2018

doi: 10.28931/riiad.2018.1.6
} 


\section{INTRODUCCIÓN}

La cannabis es la droga ilegal que más se consume en el mundo, su prevalencia anual es de 3.8\%; aproximadamente 183 millones de personas adultas usaron cannabis el último año (Oficina de las Naciones Unidas Contra la Droga y el Delito [UNODC], 2017). No es de extrañar, entonces, que la droga por la cual hay más solicitudes de tratamiento sea ésta: más de un tercio de las personas tratadas por consumo de drogas son atendidas por su uso (UNODC, 2017).

El consumo de cannabis ha aumentado y la demanda de atención sigue dicho incremento; por lo tanto, es indispensable contar con medidas de prevención y tratamiento que afronten exitosamente estas tendencias. Sin embargo, en el mundo sólo una de cada seis personas usuarias de drogas acude a tratamiento (UNODC, 2017). Transporte, costos, estigmatización y discriminación son algunos de los obstáculos, identificados por los propios usuarios de sustancias, para el acceso a los servicios de atención tradicionales (Substance Abuse and Mental Health Services Administration, 2012).

En el ámbito de la salud se ha desarrollado una amplia gama de estrategias de promoción, prevención y tratamiento, en la cual se ha tomado como base los recursos que ofrece Internet y otras tecnologías digitales, de la información y la comunicación (Gill, Gill, \& Young, 2013; Vona et al., 2014). Su implementación se puede observar en distintas áreas, por ejemplo: educación sexual (Gold et al., 2011), VIH (Jones \& Salazar, 2016), salud mental (Clarke, Kuosmanen, \& Barry, 2015); así como en el tratamiento de diferentes psicopatologías como depresión, ansiedad (Christensen, Griffiths, \& Korten, 2002) y esquizofrenia (Välimäki, Athanasopoulou, Lahti, \& Adams 2016).

Recientemente, estas herramientas se han empleado para el tratamiento de problemas relacionados con el uso y el abuso de drogas. Las intervenciones basadas en la tecnología (Litvin, Abrantes, \& Brown, 2013) se definen como un conjunto de contenidos y estrategias que -en ausencia del contacto humano directo- se transmiten o comunican por medio de una interfaz interactiva, mediante un dispositivo tecnológico (computadoras personales o dispositivos móviles). Su objetivo es promover modificaciones en el comportamiento del consumo de sustancias o en aquellos factores relacionados con éste.

Este tipo de intervenciones se caracteriza por ser de bajo costo, por la posibilidad de realizarse a distancia (tienen gran cobertura y favorecen tanto al usuario como al proveedor de los servicios), así como por sus opciones de privacidad (Barak, Klein, \& Proudfoot, 2009); se presenta como una alternativa que podría contribuir a eliminar varios de los obstáculos en el acceso a la atención por uso de drogas.
En la actualidad, existen intervenciones digitales para la atención del consumo de alcohol (Balhara \& Verma, 2014; Leeman, Pérez, Nogueira, \& DeMartini, 2015; Lehto \& Oinas-Kukkonen, 2011; Riper et al., 2011), y para la reducción del consumo de tabaco (Balhara \& Verma, 2014b; Tait \& Christensen, 2010) y de drogas ilegales, como los estimulantes anfetamínicos (Tait et al., 2015) y la cannabis.

Tait, Spijkerman y Riper (2013) Ilevaron a cabo una revisión sistemática y un metaanálisis para evaluar la efectividad de las intervenciones para la reducción del consumo de cannabis realizadas vía Internet o por computadora. En el estudio se incluyeron tratamientos e intervenciones preventivas que reportaran una medida de reducción del uso de cannabis; resultaron elegidos diez ensayos clínicos. Los estudios de la revisión tienen diversas poblaciones, intervenciones y escenarios de reclutamiento de los participantes (escuelas, clínicas, Internet, entre otros). En todos los estudios, la efectividad se midió a través de autorreportes. Los resultados arrojaron resultados significativos pero pequeños en la reducción del consumo a corto plazo $(g=.16)$. Ningún otro factor evaluado (edad, tipo de tratamiento recibido, etcétera) reveló diferencias significativas. Entre las limitaciones de esta revisión se encuentran la baja cantidad de investigaciones incluidas, que la mitad de las intervenciones se dirigieran exclusivamente a mujeres jóvenes y la fecha de los estudios, pues, en su mayoría, fueron realizados y reportados antes de 2010 . Todas estas situaciones dificultan la generalización de los resultados obtenidos.

Como ya se ha mencionado, un aspecto importante de estas intervenciones radica en la posibilidad de realizarlas fuera del contexto clínico, situación relevante si se considera que, de acuerdo con el European Monitoring Centre for Drugs and Drug Addiction (EMCDDA, 2015), nueve de cada diez usuarios problemáticos de mariguana no tienen ningún contacto con las instalaciones tradicionales para el tratamiento de las adicciones.

Con la intención de saber si las intervenciones a distancia son igual de efectivas cuando se reclutan participantes fuera del contexto clínico, Hoch, Preuss, Ferri y Simon (2016) realizaron un metaanálisis centrado en estudios, cuyo reclutamiento no hubiera sido realizado en escenarios como universidades, centros de trabajo o clínicas de atención. Incluyeron únicamente las intervenciones de tratamiento, dejando fuera las de prevención, sin justificar la exclusión. El análisis recopiló información de cuatro estudios y sus resultados mostraron efectos pequeños en la reducción del consumo de cannabis, menores que los registrados por Tait et al. (2013), pero estadísticamente significativos (ES = .11).

Una de las principales limitaciones de ambos trabajos radica en la inclusión de pocos estudios. En el segundo 
caso, es llamativo que se incluya menos de la mitad de estudios que en el referido en primer lugar. Una posible explicación para esta diferencia podría hallarse en dos restricciones que el trabajo de Hoch et al. (2016) no comparte con el primero: la relacionada con el reclutamiento en escenarios extraclínicos y la exclusión de intervenciones preventivas. Los resultados del metaanálisis de Tait et al. (2013) no permiten justificar las restricciones que hace Hoch et al. (2016), pues no reflejan diferencias significativas en cuanto a intervenciones preventivas y de tratamiento ni de ningún posible moderador. Sobre el reclutamiento, el concentrarse en escenarios extraclínicos excluye muchos ensayos que se sirven de los sistemas educativos o de salud para llamar a los participantes; además, el estudio no diferencia lo suficiente a la población que trata de especificar, ni justifica teóricamente la posible mayor efectividad de una población en comparación con otras.

Se puede resumir que el estudio conducido por Tait et al (2013) incluyó artículos realizados y reportados hace más de siete años y que el de Hoch et al. (2016) mantiene criterios muy estrictos no justificados y que incorpora a su análisis muy pocos estudios.

Además de estas revisiones citadas y comentadas anteriormente, no existen trabajos sobre las características ni la efectividad de las intervenciones digitales para la reducción del consumo de cannabis. Por lo anterior, se plantea la necesidad de realizar una revisión sistemática acerca de la efectividad de las intervenciones digitales actuales para la reducción del consumo de cannabis, que hayan empleado ensayos clínicos aleatorizados, tanto de prevención como de tratamiento, en escenarios clínicos y extraclínicos, siempre y cuando ofrezcan resultados de la reducción del consumo en términos de las anteriores revisiones. En la presente revisión se entenderá por efectividad, la reducción del consumo de cannabis estadísticamente significativa.

El objetivo de esta revisión se cumple con la síntesis cualitativa presentada; queda pendiente la síntesis cuantitativa o metaanálisis.

\section{MÉTODO}

\section{Localización y selección de fuentes bibliográficas potenciales}

La búsqueda se llevó a cabo en octubre 2017 y febrero del 2018 en el Cochrane Central Register of Controlled Trials (que contiene registros provenientes de Medline y EMBASE, actualizados cada tres y doce meses respectivamente), así como PsycINFO y PubMed. En las tres fuentes se empleó el mismo intervalo de búsqueda (2010-2017, 2018) y no hubo restricción de idioma. Las palabras usadas fueron una combinación de términos MeSH y texto libre, que se eligieron en referencia a las revisiones sobre el mismo tema (Hoch et al., 2016; Litvin et al., 2013; Tait et al., 2013).

Se emplearon las siguientes palabras y términos: 1) substance related disorders OR addiction OR abuse OR dependence OR illicit AND; 2) cannabis OR marihuana OR marijuana OR hashish AND 3) Internet OR web OR online OR computer OR cd rom OR digital OR cyber OR virtual reality OR cell phone OR SMS OR text message OR texting OR e-health OR app AND; 4) treatment OR intervention $O R$ prevention.

Se realizó también una búsqueda manual intencionada de acuerdo con las referencias de los estudios que cumplieron con los criterios de elegibilidad.

\section{Criterios de inclusión y exclusión}

Se incluyeron los artículos que cumplieron los siguientes requisitos:

1. Ensayos clínicos controlados aleatorizados.

2. Que evaluaran el efecto de una intervención realizada vía Internet o computadora para la prevención o el tratamiento del consumo de cannabis, en particular, o de drogas, en general.

3. Que una de sus medidas de resultado fuese la variación en el uso de mariguana.

Se excluyeron artículos de acuerdo con los siguientes criterios:

1. Que sólo presentaran resultados sobre conocimientos teóricos, actitudes o intenciones de consumir mariguana, sin incluir una medida en la variación del consumo.

2. Reportes de investigaciones que evaluaran la reducción del consumo de drogas en general, sin presentar una medida en específico sobre la variación del consumo de mariguana.

3. Investigaciones que hayan evaluado intervenciones para la reducción del consumo de alguna droga diferente a la cannabis.

4. Artículos que ofrecieran resultados sobre una muestra analizada en ensayos previamente considerados en el presente estudio.

5. Textos que presentaran resultados que fueron actualizados en un seguimiento posterior. En esos casos se incluyó el artículo que presentase el seguimiento más reciente.

6. Informes que hicieran referencia a ensayos publicados o que no contasen con información suficiente sobre el estudio reportado. 


\section{Evaluación del riesgo de sesgo de los estudios incluidos}

La evaluación del riesgo de sesgo en los estudios elegidos se hizo de acuerdo con los criterios de la Colaboración Cochrane (Centro Cochrane Iberoamericano, 2012) y con ayuda del software RevMan 5.3 (Nordic Cochrane Centre Review Manager, 2014). En cada uno de los estudios se evaluaron los siguientes criterios: sesgo de selección, de realización y de desgaste.

\section{RESULTADOS}

La búsqueda emprendida produjo 1022 registros, de los cuales 26 cumplieron con los criterios de inclusión. Los detalles se describen en el diagrama de flujo de selección de estudios (Figura 1).
Las características de los ensayos se presentan en la Tabla 1, de descripción de ensayos. Se tomó como base la caracterización realizada en los estudios de Hoch et al. (2016) y Tait et al. (2013): cada fila presenta información de los ensayos seleccionados, ordenados por el año de publicación. Las categorías incluidas fueron:

1. Primer autor, año de publicación, país en el que se llevó a cabo la intervención.

2. Participantes: si una característica relevante constituía una variable (sexo, edad, grado escolar, condición psiquiátrica, entre otras), en algunos casos se convertía en un puntaje en algún instrumento como el Inventario de Depresión de Beck BDI-II o el Alcohol, Smoking and Substance Involvement Screening Test (ASSIST). Se anotó media de edad

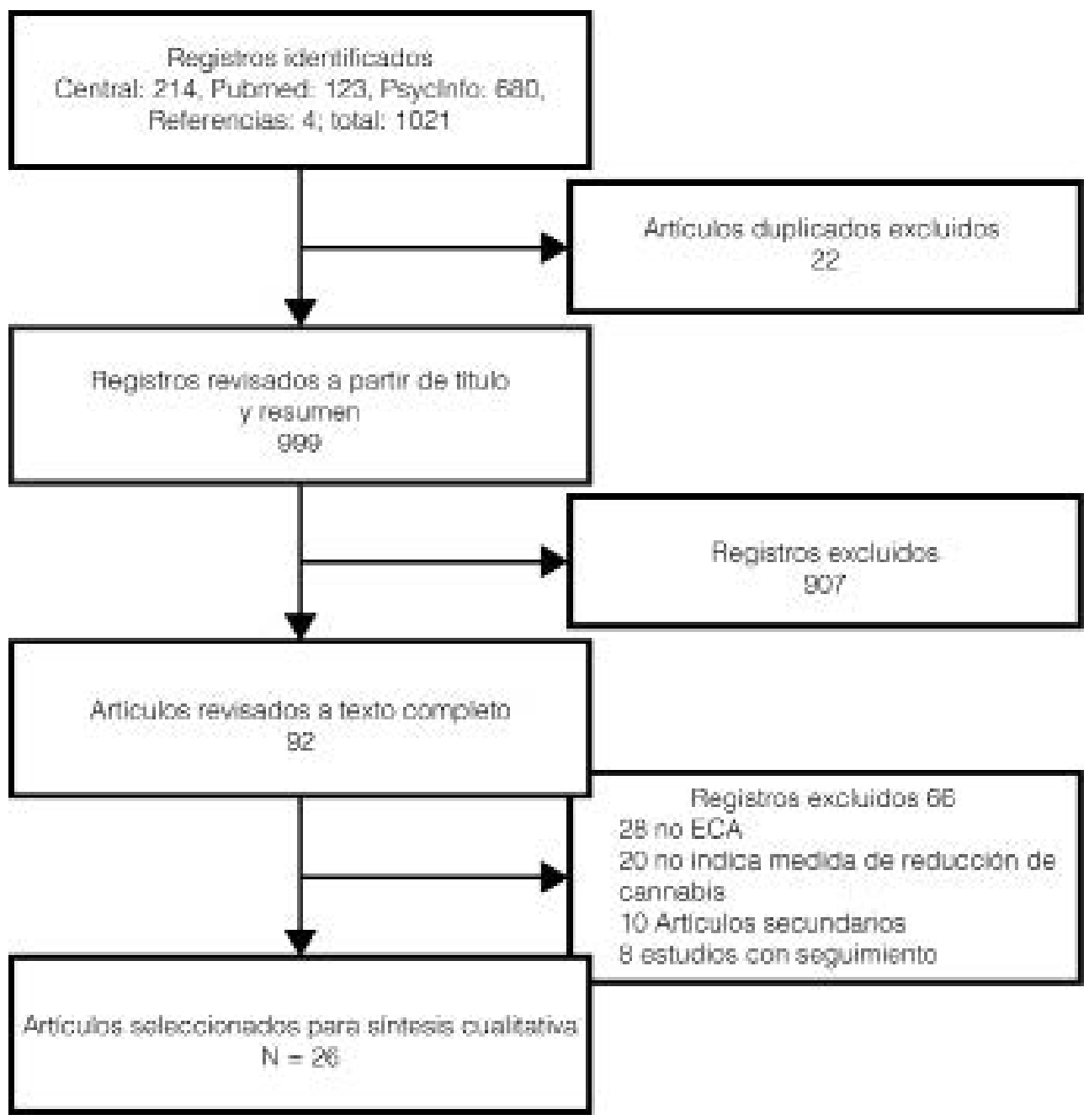

Figura 1. Diagrama de flujo de proceso de selección de estudios 
$(\bar{X})$, número de participantes en la muestra (N) y número específico de participantes consumidores de mariguana en la muestra total, si se trataba de un estudio sobre varias drogas de consumo.

3. Modo de entrega (escenario): si la intervención se desarrolló en un lugar específico como clínicas o escuelas, si se realizó a través de un sitio web y si se empleó algún dispositivo tecnológico particular como tabletas o teléfonos inteligentes.

4. Intervención, comparación: se anotó en cursivas el nombre de la intervención (IT), si se mencionaba; alguna característica relevante como el enfoque teórico de la misma, el tipo de control o comparación (C) y si la intervención era denominada como preventiva o de tratamiento. También se usaron las siguientes abreviaturas: entrevista motivacional (EM), terapia de incremento motivacional (TIM), terapia cognitivo conductual (TCC), intervención breve (IB) y terapia centrada en la persona (TCP).

5. Duración de la intervención: días, minutos (min), sesiones (S), módulos u otra información disponible al respecto.

6. Si había algún tipo de guía o facilitación por otra persona $(G)$ o si era automatizada (A).

7. La medida de uso de mariguana (M) empleada y el momento posterior a la intervención en que se llevaron a cabo el o los post-test (P). Se indicó también si se usó algún instrumento de autorreporte para la medición, por ejemplo Opiate Treatment Index (OTI Q) o Add Health, o si se usó prueba biológica, en ese caso se escribió Mpb.

8. Resultado: siempre se refirió exclusivamente a la reducción del uso de cannabis.

9. Retención (R): el porcentaje de retención de participantes en el último seguimiento.

Las revisiones de este tipo de intervenciones suelen reunir estudios que varían ampliamente en sus características: población, enfoque teórico y duración, entre otras (Barak et al., 2009; Litvin et al., 2013; Strecher, 2007). De igual forma, esta revisión encontró una gran variación en categorías como edad, participantes y enfoque teórico, etcétera.

En Estados Unidos de América se ha concentrado la mayor parte de los estudios al respecto, 14 de los ensayos seleccionados se realizaron ahí; en Australia se hicieron seis. En Alemania, Países Bajos, Suiza y Canadá también se han desarrollado y probado intervenciones digitales para reducir el consumo de mariguana mediante ensayos clínicos aleatorizados.

Los participantes de los estudios seleccionados variaron en diferentes características. Poco más de la mitad de los ensayos se realizó con adultos y se encontraron diez con participantes de entre once y 18 años edad.
En cinco estudios, la muestra se conformó únicamente por mujeres. Otras condiciones de los participantes fueron: mujeres adultas postparto, presencia de comorbilidad (específicamente depresión), diadas madre-hija, estudiantes universitarios, usuarios problemáticos de mariguana, personas con tabaquismo, entre otras.

En relación al escenario o modo de entrega se encontró que en la mitad de los estudios (13) el escenario fue una página de Internet; sin contar otros en los que se desarrolló de manera combinada en Internet y en una escuela o clínica.

Los ensayos que combinaron las formas de entrega o que solicitaban que el participante se trasladara a una escuela o clínica fueron intervenciones breves, de tipo consejería, con aplicación en sitios y momentos específicos, como en visitas a servicios médicos (Gryczynski, O'Grady, Schwartz, Mitchell, \& Ondersma, 2016; Ondersma et al., 2014; Walton et al., 2013; Walton et al., 2014) o en centros escolares (Lee et al., 2010). Algunas otras eran intervenciones más complejas que tenían varios componentes y que requerían, incluso, la presencia del participante (por ejemplo Budney et al., 2015; Cochran et al., 2015) para desarrollar el manejo de contingencias. Otros, como el conducido por Newton et al. (2010), incluían un componente que pedía la realización de actividades grupales dirigidas por el profesor. Algunos pocos ensayos hicieron uso de tabletas electrónicas (Blow et al., 2015; Ondersma et al., 2014).

Se ubicaron diferentes técnicas y enfoques teóricos, a saber: entrevista motivacional, terapia de incremento motivacional, terapia cognitivo conductual, manejo de contingencias, reforzamiento social, consejería, teoría de la interacción familiar, teoría de la influencia social y terapia centrada en soluciones. De las anteriores, la más frecuente fue la entrevista motivacional, como un componente más de la intervención (por ejemplo Schaub et al., 2015) o como la intervención principal (Jonas et al., 2012); en el último caso, siempre se trató de intervenciones breves. También se usó una terapéutica derivada de la entrevista motivacional, la terapia de incremento motivacional. Otros enfoques frecuentes fueron el cognitivo conductual y la teoría de la interacción familiar, que fueron la base de cuatro intervenciones.

En los ensayos seleccionados se encontraron nueve intervenciones preventivas, seis de ellas tuvieron una duración de seis o más sesiones. En contraste, se identificaron intervenciones breves de pocos minutos, por ejemplo las de Gryczynski et al. (2016) o Jonas et al. (2012), de 30 minutos o menos. En total, se contabilizaron once intervenciones de una sesión; las demás variaron entre seis y 12 sesiones. Cochran et al. (2015) reportó una intervención de 62 módulos; sin embargo, su duración en tiempo fue también de 12 semanas. 


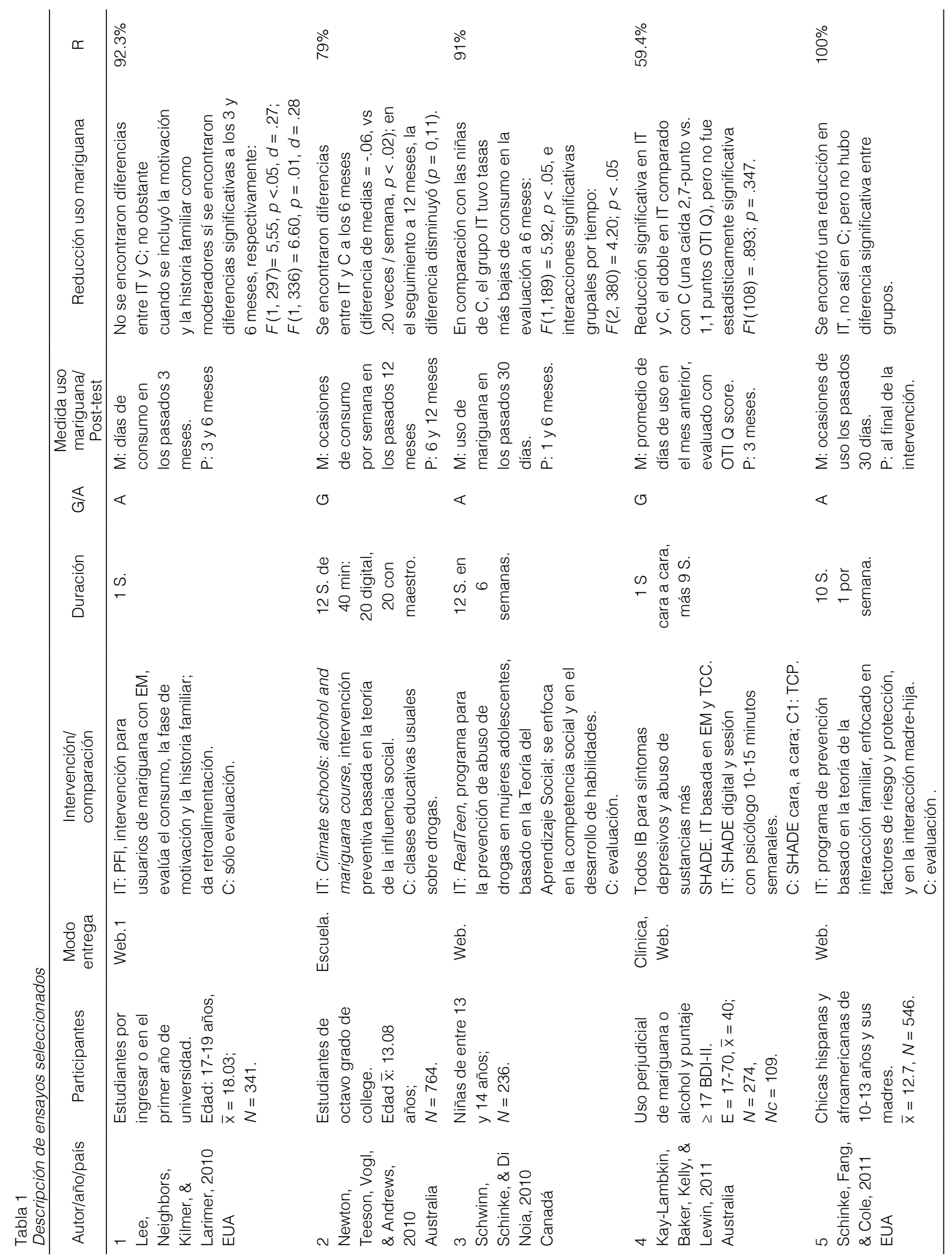




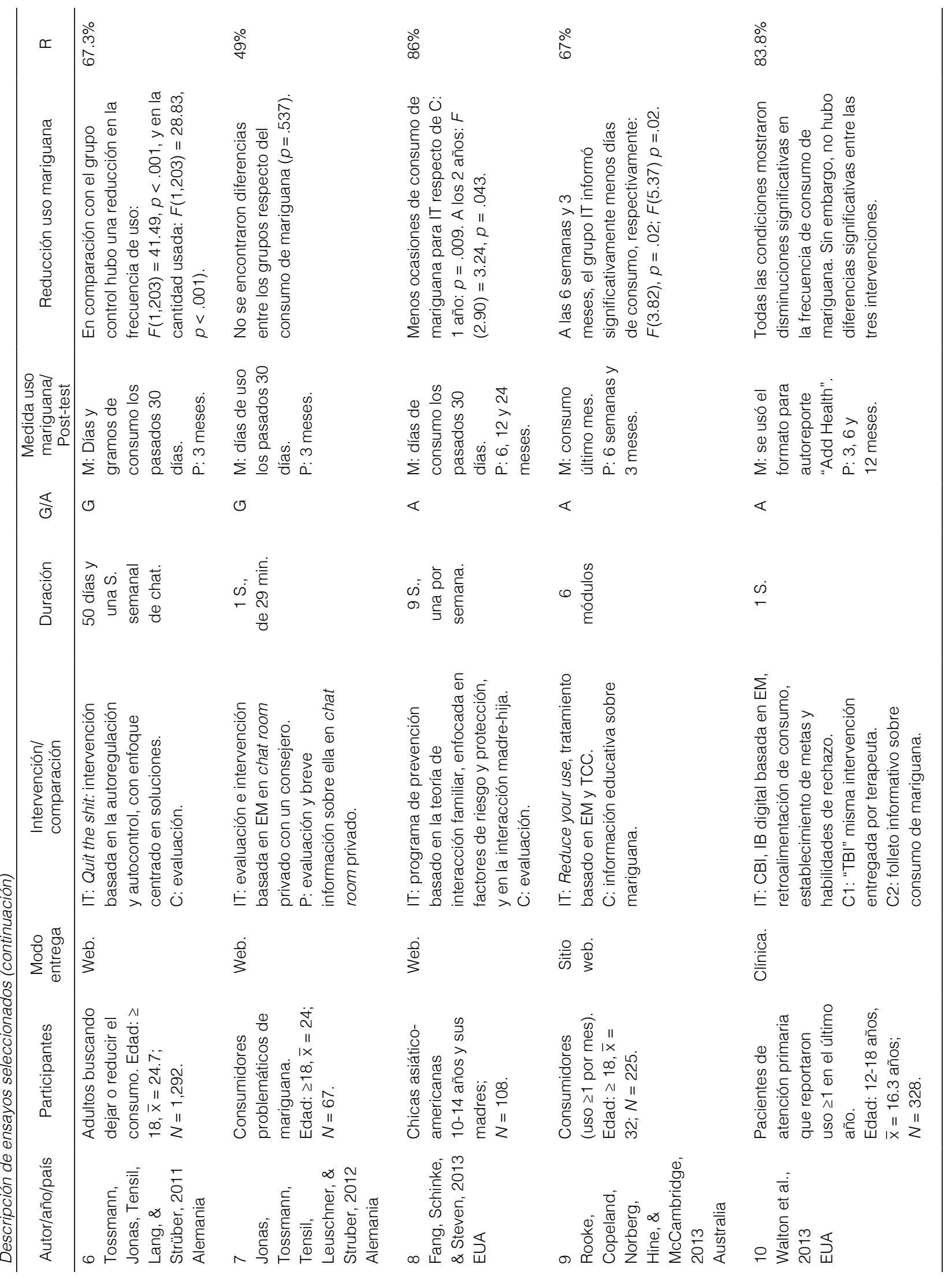




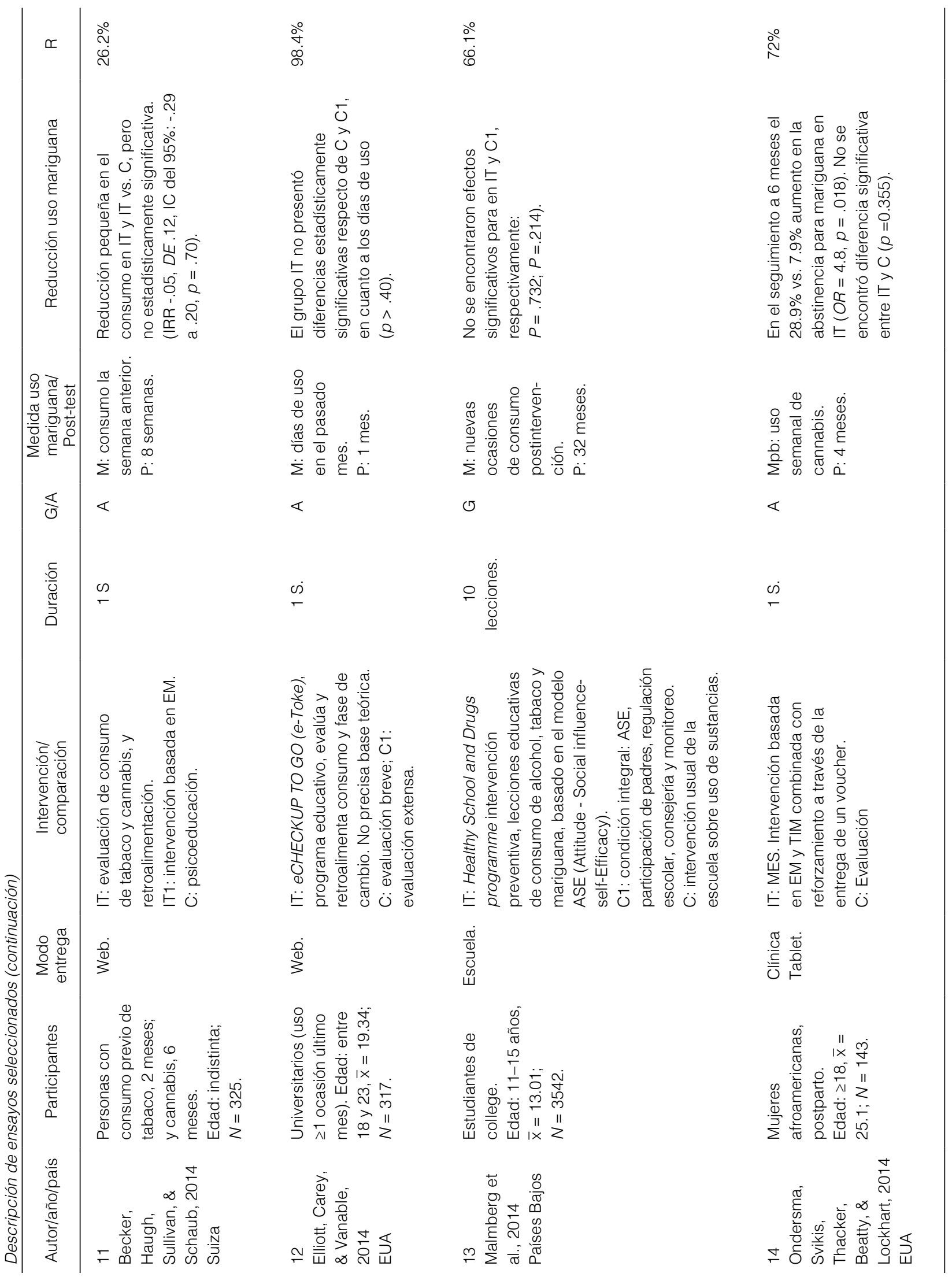




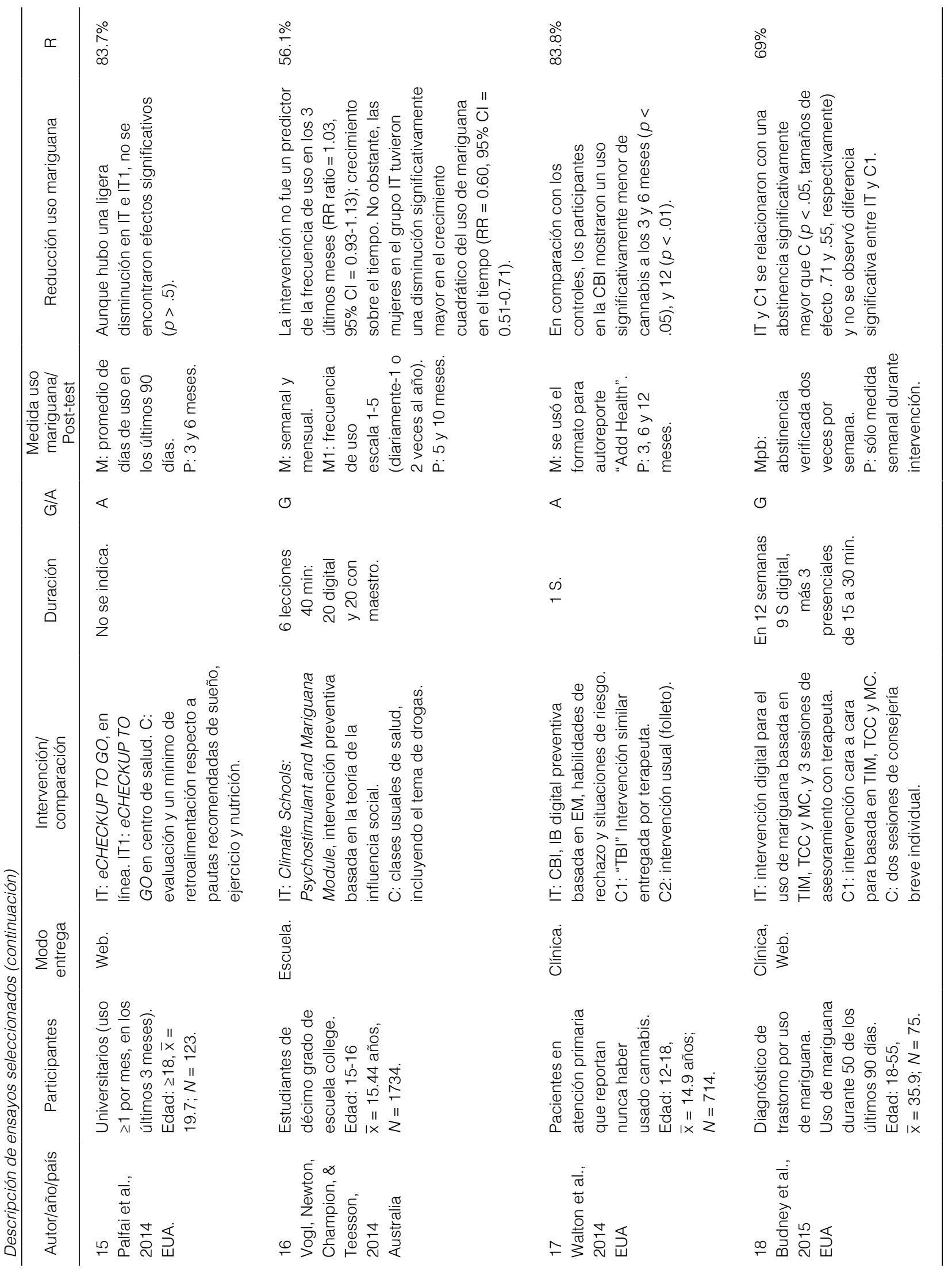




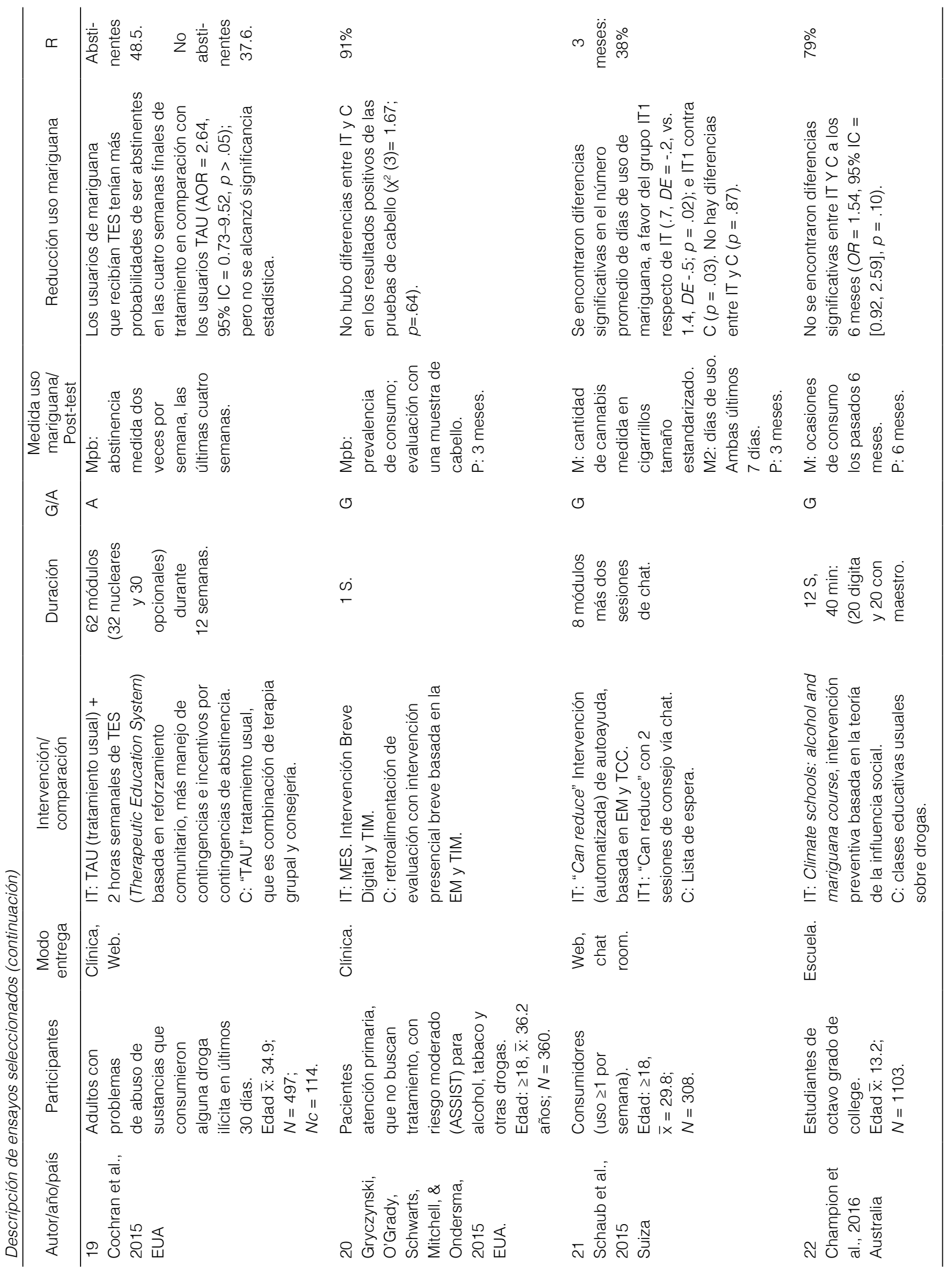




\begin{tabular}{|c|c|c|c|c|}
\hline$\simeq$ & $\begin{array}{l}\stackrel{0}{\circ} \\
\infty \\
\infty\end{array}$ & ○ें & $\begin{array}{l}\circ \\
\circ \\
0\end{array}$ & 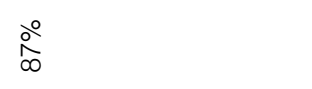 \\
\hline 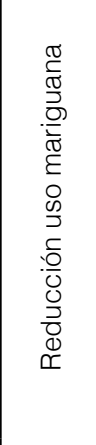 & 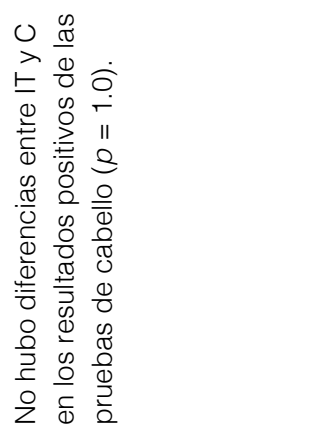 & 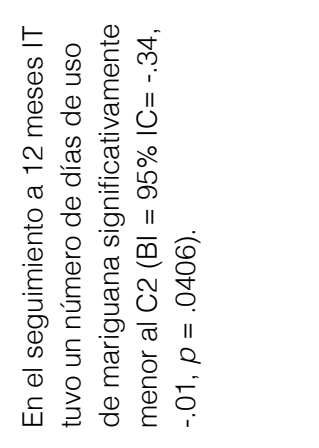 & 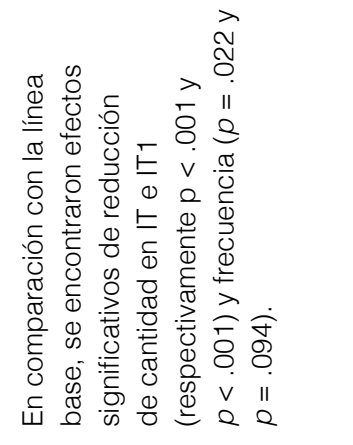 & 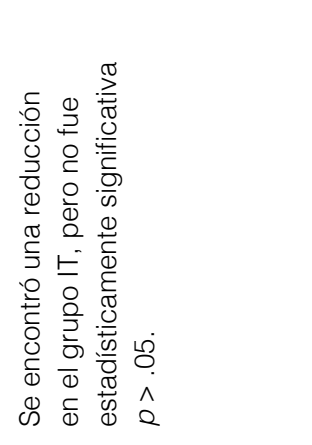 \\
\hline 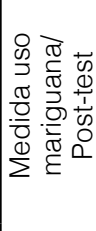 & 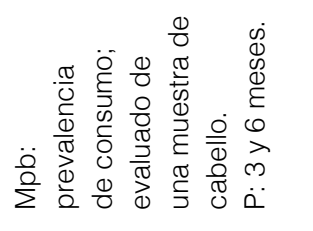 & 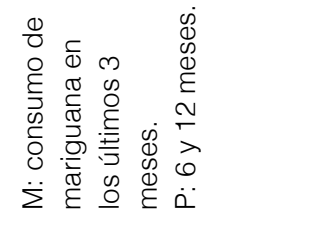 & 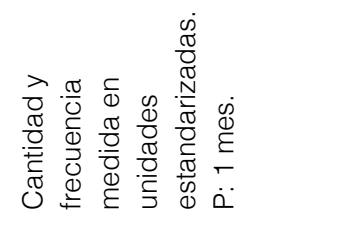 & 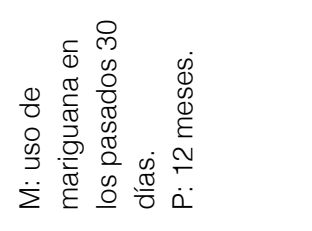 \\
\hline$\frac{\pi}{\Delta}$ & $\varangle$ & $\varangle$ & $\varangle$ & $\varangle$ \\
\hline 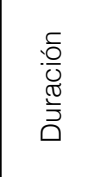 & 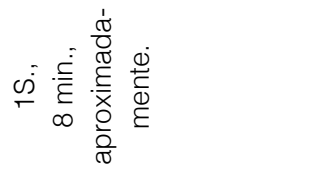 & $\stackrel{\dot{\omega}}{\ulcorner}$ & $\stackrel{\dot{c}}{-}$ & 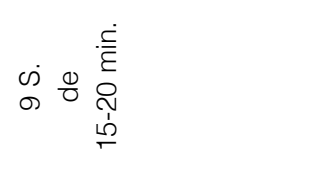 \\
\hline 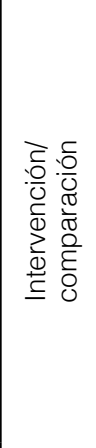 & 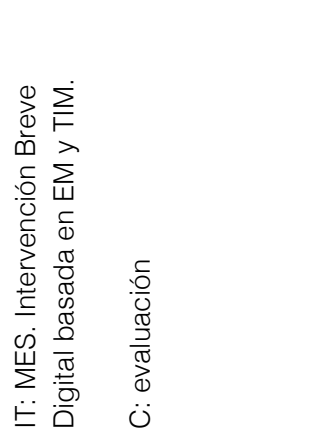 & 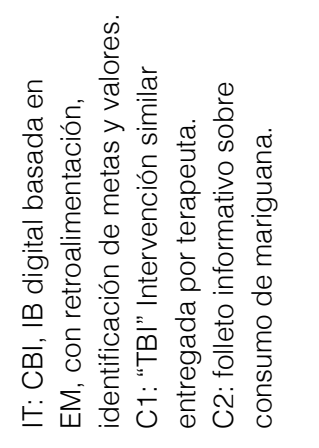 & 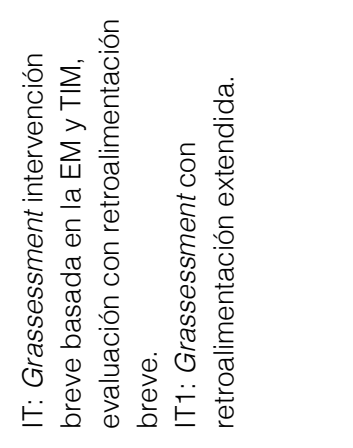 & 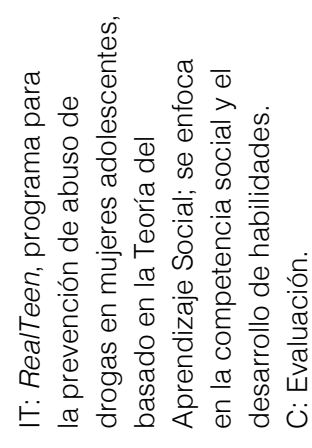 \\
\hline 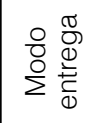 & 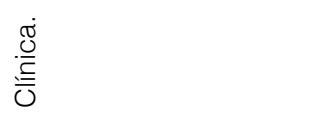 & 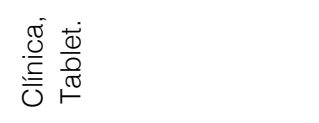 & $\frac{\dot{\theta}}{3}$ & $\frac{\dot{0}}{3}$ \\
\hline 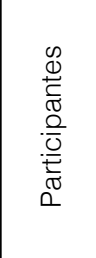 & 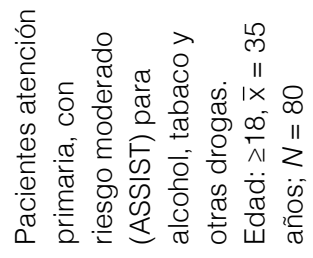 & 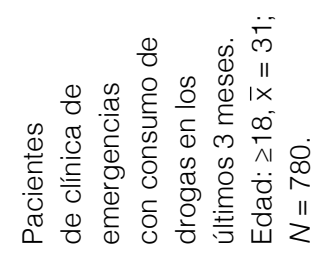 & 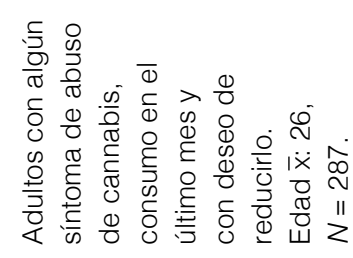 & 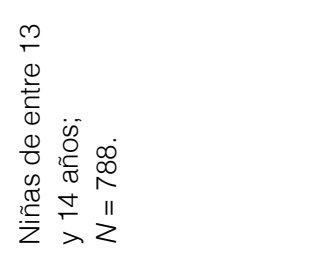 \\
\hline 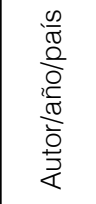 & 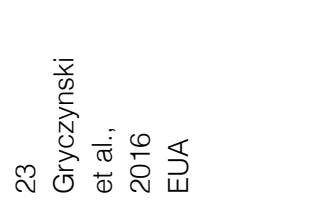 & 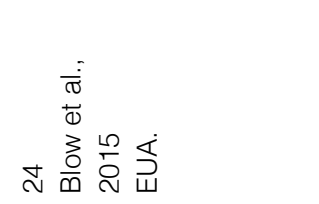 & 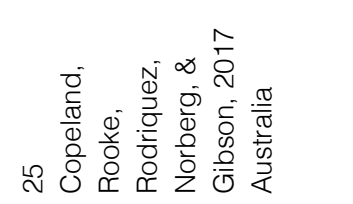 & 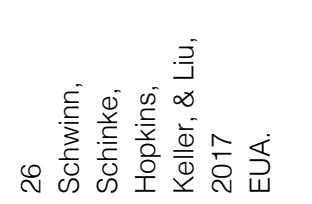 \\
\hline
\end{tabular}


La autoadministración es una de las grandes peculiaridades de las intervenciones digitales, en este rubro se encontró que un poco más de la mitad de los ensayos (16) reportó intervenciones automatizadas; las demás requirieron una participación variable de un especialista.

Se emplearon diferentes medidas de uso de mariguana para evaluar la efectividad. En primer término se distinguieron tres tipos de instrumentos: el autorreporte fue el más frecuente (por ejemplo en Fang et al., 2013; Rooke et al., 2013; Schwinn et al., 2010); también se usaron instrumentos estandarizados como OTI Q (Kay-Lambkin et al., 2011) o Add Health (Walton et al., 2013); así como muestras biológicas -recogidas en siete estudios-, principalmente de orina (Budney et al., 2015; Cochran et al., 2015; Ondersma et al., 2014) y cabello (Gryczynski et al., 2015).

Se encontraron también diferentes periodos para reportar el consumo: diario, semanal, último mes y últimos tres meses. Destacó el estudio de Tossman et al. (2011) en el que se pidió a los participantes llevar un registro diario del consumo, y el de Schaub et al. (2015) que, al inicio de la intervención, tuvo un procedimiento para estandarizar la medida de la cantidad de consumo en los participantes, quienes especificaban si habían consumido mariguana o hachís, si habían usado una pipa o un cigarrillo y el tamaño del mismo, de tal manera que fuera posible calcular, con mayor precisión, la cantidad consumida.

En la mitad de los registros (13) se pudo observar un efecto de reducción estadísticamente significativo del consumo de cannabis, asociado con la intervención digital y contrastado con al menos uno de los controles o comparaciones. En ocho casos, dichos controles fueron únicamente de evaluación; en los restantes, se comparó con intervenciones cara a cara o con tratamientos usuales. De estos casos sólo se encontraron dos en los que la intervención digital superó los resultados de la realizada cara a cara (Budney et al., 2015; Walton et al., 2014).

Se observó que casi dos terceras partes de los ensayos tuvieron porcentajes de retención de arriba de 70\%; la mitad de éstos, más de $90 \%$. Tres estudios tuvieron menos de $50 \%$ : entre 35 y $45 \%$.

En la evaluación del riesgo de sesgo (véase Figura 2) se encontró que hay un alto porcentaje de bajo riesgo en el sesgo de selección, ello debido, en parte, a que uno de los criterios de selección fue el que los estudios fueran ensayos aleatorizados; a pesar de ello, en algunos casos la asignación no fue clara. Los otros tipos de sesgo tuvieron mayor porcentaje de riesgo alto o de poco claro; especialmente en el sesgo de realización, debido quizá a las dificultades que los ensayos tuvieron para incluir en su procedimiento el cegamiento del personal colaborador, más que el de los participantes; es cierto también que la falta de cegamiento no necesariamente implica un alto riesgo. En el caso del sesgo de desgaste, casi todos los estudios emprendieron análisis de intención de tratar; no obstante, hubo altas tasas de desgaste en muchos de ellos.

\section{DISCUSIÓN Y CONCLUSIONES}

Los 26 estudios incluidos evaluaron 16 intervenciones diferentes para la reducción del consumo de cannabis; es decir, más del doble que en Tait et al. (2013) y mucho más que en Hoch et al. (2016). Este resultado muestra tanto la necesidad de actualizar las revisiones precedentes como la pertinencia del presente estudio.

Las intervenciones encontradas se han desarrollado y probado en apenas unos pocos países; todos ellos

Gereración de la secuencin aleatona (sesgo de selección) Ocultanjento de la arignación (sesgo de selección) Cegamiento de participantes y personal (sesgo de realización) Manejo de los datos de resultado incompletos (sesgo de desgaste)

Otros sesgos

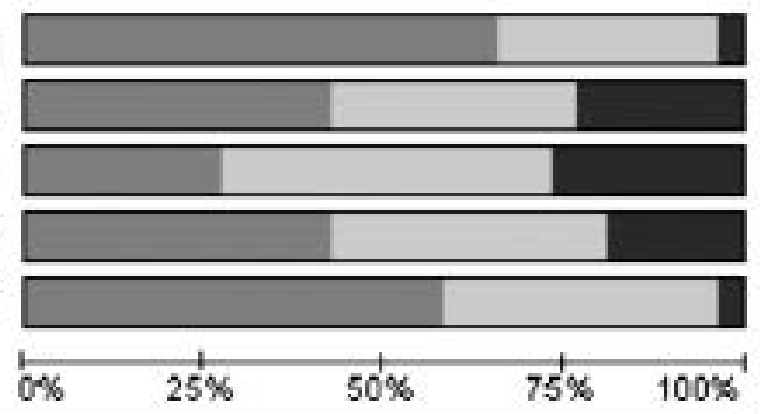

\section{Bajo riesgo de sesgo}

Poco claro riesgo de sesgo

Alto riesgo de sesgo

Gráfico de riesgo de sesgo: presenta el porcentaje total de riesgo para cada elemento evaluado individualmente en cada estudio incluido en la revisión.

Figura 2. Gráfico de riesgo de sesgo 
con un alto consumo de cannabis y prevalencias de más de 8\% (consumo el último año); excepto Alemania y Suiza, cuya prevalencia se mueve entre 6 y $8 \%$ (UNODC, 2013).

Gran parte de las intervenciones hacen uso de Internet y muchas de ellas se desarrollan en sitios web. El sitio web es propiamente un espacio, un escenario donde se desarrolla la intervención y sus características pueden ser relevantes para que la intervención sea efectiva (Barak, et al., 2009): el que el participante pueda desempeñarse en el lugar que elija es un elemento importante de personalización, una cualidad que, considerando las dificultades de acceso al tratamiento, podría contribuir a disminuir estos obstáculos. En relación a ello, cabe destacar que sólo en dos ensayos se usó tecnología móvil (tablets) para la entrega de la intervención, y sólo se encontró una aplicación (app) con fines psicoterapéuticos (Rooke et al., 2015) para la reducción del consumo de cannabis (no se incluyó en la presente revisión debido al diseño del estudio). En una búsqueda previa, Ramo, Popova, Grana, Zhao y Chavez (2015) tampoco encontraron apps de intervenciones de tratamiento para usuarios de cannabis.

Además de la movilidad, otra de las grandes particularidades de las intervenciones digitales es la posibilidad de automatización, característica de más de la mitad de las intervenciones en esta revisión. La entrevista motivacional, la terapia de incremento motivacional, así como la terapia cognitivo conductual son técnicas y enfoques teóricos usuales en el tratamiento para las adicciones, ya sea basado o no en la tecnología (Becoña et al., 2008; EMCDDA, 2015b), que han sido adaptados a versiones digitales y automatizados (Shingleton \& Palfai, 2016).

De igual forma, dichas terapéuticas son o tienen versiones breves de hasta una sesión de pocos minutos, especialmente la entrevista motivacional; no parece ser casualidad entonces que casi la mitad de los ensayos hayan practicado intervenciones de una sola sesión.

Ninguna característica de los participantes (edad, sexo) o de las intervenciones (duración, enfoque teóri$\mathrm{co}$, automatización o cualquier otra) parece ser relevante para la efectividad en la reducción del consumo y ninguna de ellas es constante en las intervenciones efectivas o en las que no lo son. El panorama que ofrece la revisión no alcanza a señalar cuáles son los moderadores que delinean las mejores intervenciones. Previamente se ha señalado la dificultad para determinar las cualidades de las intervenciones digitales efectivas (Strecher, 2007); ello se ha debido, al menos en parte, a la diversidad de éstas y a que las comparaciones hechas en revisiones o metaanálisis previos han emprendido dicha tarea soslayando esta diversidad, al suponer que tienen lo suficiente en común, por sobre sus diferencias (Litvin et al., 2013). A esto se suma el hecho de que también se deja de lado la importancia de las características propiamente digitales, tanto que pocos estudios se ocupan en describir con detalle esas peculiaridades (Barak et al., 2009; Tiburcio et al., 2016).

Existen intervenciones digitales efectivas para la reducción del consumo de cannabis. Se requiere mayor investigación para determinar la población, los escenarios y las características específicas en las que éstas los son. En especial se requiere investigación que considere las características que les son propias como intervenciones digitales; entre ellas la automatización, la movilidad y los sitios web como auténticos escenarios virtuales.

Al respecto de una de estas características, la movilidad, se encontró que hay muy poco uso de dispositivos móviles para la entrega y el desarrollo de estas intervenciones. La mayoría de ellas se han diseñado y probado en países con alto consumo de cannabis; no obstante, teniendo en cuenta la demanda tratamiento, así como las dificultades reportadas para acceder a éste, es de esperarse que las intervenciones digitales se desarrollen y apliquen con frecuencia.

\section{Alcances y limitaciones}

El presente estudio identificó las intervenciones para la reducción del uso cannabis que han sido probadas mediante ensayos clínicos aleatorizados, lo anterior mediante una exhaustiva búsqueda en dos de las más grandes bases de datos sobre salud y en una de las más importantes sobre temas psicológicos. La cantidad de estudios encontrados, en comparación con las revisiones previas sobre el mismo tema, evidencia la pertinencia de la empresa; no obstante, la disparidad entre las intervenciones incluidas imposibilita una evaluación precisa de la eficacia de las mismas, como la que podría aportar un metaanálisis. Otra limitación importante es que ninguno de los procedimientos se llevó a cabo por pares, como recomienda la Colaboración Cochrane, lo que aumenta el riesgo de sesgo de los resultados de esta revisión.

\section{FINANCIAMIENTO}

El presente estudio es un producto del Programa de Investigación de Centros de Integración Juvenil, A.C. No se recibió financiamiento específico para su desarrollo.

\section{CONFLICTOS DE INTERÉS}

El autor forma parte de Centros de Integración Juvenil, A. C., no obstante el contenido del presente estudio representa su opinión, no la de la organización. 


\section{REFERENCIAS}

Balhara, Y. P. S., \& Verma, R. (2014). A review of web based interventions focusing on alcohol use. Annals of Medical and Health Sciences Research, 4(4), 472-480. doi: 10.4103/2141-9248. 139272

Balhara, Y. P. S., \& Verma, R. (2014b). A review of web based interventions for managing tobacco use. Indian Journal of Psychological Medicine, 36(3), 226-235. doi: 10.4103/0253-7176.135367

Barak, A., Klein, B., \& Proudfoot, J. G. (2009). Defining Internet-supported therapeutic interventions. Annals of Behavioral Medicine, 38(1), 4-17. doi: 10.1007/s12160-009-9130-7.

Becker, J., Haug, S., Sullivan, R., \& Schaub, M. P. (2014). Effectiveness of different web-based interventions to prepare cosmokers of cigarettes and cannabis for double cessation: a three-arm randomized controlled trial. Journal of Medical Internet Research, 16(12), e273. doi:10.2196/jmir.3246

Becoña, I. E., Cortés, T. M. T., Pedrero, P. E. J., Fernández, H. J. R., Casete, F. L., Bermejo, G. M. P., ... Tomás, G. V. (2008). Guía clínica de intervención psicológica en adicciones. Barcelona: Socidrogalcohol.

Blow, F., Bohnert, A. S., Ignacio, R., Barry, K., Ilgen, M. A., Booth, B. M.,... Walton, M. (2015). Efficacy of computer and therapist brief interventions for drug users. Drug and Alcohol Dependence, 156(1-2), 74-79. doi: 10.1016/j.drugalcdep.2015.07.975

Budney, A. J., Stanger, C., Tilford, J. M., Scherer, E., Brown, P. C., Li, Z., ... Walker, D. (2015). Computer-assisted behavioral therapy and contingency management for cannabis use disorder. Psychology of Addictive Behaviors: Journal of the Society of Psychologists in Addictive Behaviors, 29(3), 501-511. doi: 10.1037/ adb0000078

Centro Cochrane Iberoamericano, (2012). Manual Cochrane de Revisiones Sistemáticas de Intervenciones versión 5.1.0 (actualizada en marzo de 2011). Barcelona: Autor. Recuperado de http:// www.cochrane.es/?q=es/node/269

Champion, K. E., Newton, N. C., Stapinski, L., Slade, T., Barrett, E. L., \& Teesson, M. (2016). A cross-validation trial of an Internet-based prevention program for alcohol and cannabis: preliminary results from a cluster randomised controlled trial. Australian \& New Zealand Journal of Psychiatry, 50(1), 64-73. doi: 10.1177/0004867415577435

Christensen, H., Griffiths, K. M., \& Korten, A. (2002). Web-based cognitive behavior therapy: analysis of site usage and changes in depression and anxiety scores. Journal of Medical Internet Research, 4(1), e3.

Clarke, A. M., Kuosmanen, T., \& Barry, M. M. (2015). A systematic review of online youth mental health promotion and prevention interventions. Journal of Youth and Adolescence, 44(1), 90-113. doi: 10.1007/s10964-014-0165-0.

Cochran, G., Stitzer, M., Campbell, A. N., Hu, M. C., Vandrey, R., \& Nunes, E. V. (2015). Web-based treatment for substance use disorders: differential effects by primary substance. Addictive Behaviors, 45, 191-194. doi: 10.1016/j.addbeh.2015.02.002
Elliott, J. C., Carey, K. B., \& Vanable, P. A. (2014). A preliminary evaluation of a web-based intervention for college marijuana use. Psychology of Addictive Behaviors, 28(1), 288. doi oi:10.1037/ a0034995

European Monitoring Center for Drugs and Drug Addiction. (2015). Annual report: the state of the drugs problem in Europe. Lisbon, Luxembourg: Publications Office of the European Union. doi:10.2810/084165

European Monitoring Centre for Drugs and Drug Addiction. (2015b). Treatment of cannabis-related disorders in Europe. Luxembourg: Publications Office of the European Union. doi:10.2810/621856

Fang, L., Schinke, S. P., Steven, P. (2013). Two-year outcomes of a randomized, family-based substance use prevention trial for Asian American adolescent girls. Psychology of Addictive Behaviors: Journal of the Society of Psychologists in Addictive Behaviors, 27(3), 788-798. doi: 10.1037/a0030925

Gill, H. K., Gill, N., \& Young, S. D. (2013). Online technologies for health information and education: a literature review. Journal of Consumer Health on the Internet, 17(2), 139-150. doi: 10.1080/15398285.2013.780542

Gold, J., Pedrana, A. E., Sacks-Davis, R., Hellard, M. E., Chang, S., Howard, S., ... Stoove, M. A. (2011). A systematic examination of the use of online social networking sites for sexual health promotion. BMC Public Health, 11(1), 583. doi: 10.1186/1471-2458$11-583$

Gryczynski, J., Mitchell, S. G., Gonzales, A., Moseley, A., Peterson, T. R., Ondersma, S. J., ... Schwartz, R. P. (2015). A randomized trial of computerized vs. in-person brief intervention for illicit drug use in primary care: outcomes through 12 months. Journal of Substance Abuse Treatment, 50, 3-10. doi: 10.1016/j. jsat.2014.09.002

Gryczynski, J., O'Grady, K. E., Schwartz, R. P., Mitchell, S. G., \& Ondersma, S. J. (2016). Immediate versus delayed computerized brief intervention for illicit drug misuse. Journal of Addiction Medicine, 10(5), 344-351.

Hoch, E., Preuss, U. W., Ferri, M., \& Simon, R. (2016). Digital interventions for problematic cannabis users in non-clinical settings: findings from a systematic review and meta-analysis. European Addiction Research, 22(5), 233-242. doi:10.1159/000445716

Jonas, B., Tossmann, P., Tensil, M., Leuschner, F., \& Struber, E. (2012). Efficacy of a single-session online-intervention on problematic substance use. Sucht, 58, 173-182. doi: 10.1024/09395911.a000182

Jones, J., \& Salazar, L. F. (2016). A review of HIV prevention studies that use social networking sites: implications for recruitment, health promotion campaigns, and efficacy trials. AIDS and behavior, 20(11), 2772-2781. doi: 10.1007/s10461-016-1342-9

Kay-Lambkin, F. J., Baker, A. L., Kelly, B., \& Lewin, T. J. (2011). Clinician-assisted computerized versus therapist-delivered treatment for depressive and addictive disorders: Aarandomized controlled trial. Medical Journal of Australia, 195(3), 44-50.

Lee, C. M., Neighbors, C., Kilmer, J. R., \& Larimer, M. E. (2010). A brief, web-based personalized feedback selective interven- 
tion for college student marijuana use: a randomized clinical trial. Psychology of Addictive Behaviors: Journal of the Society of Psychologists in Addictive Behaviors, 24(2), 265-273. doi: 10.1037/a0018859

Leeman, R. F., Pérez, E., Nogueira, C., \& DeMartini, K. S. (2015). Very-brief, web-based interventions for reducing alcohol use and related problems among college students: a review. Frontiers in P|sychiatry, 6 (129). doi: 10.3389/fpsyt.2015.00129

Lehto, T., \& Oinas-Kukkonen, H. (2011). Persuasive features in web-based alcohol and smoking interventions: a systematic review of the literature. Journal of Medical Internet Research, 1(3), e46. doi:10.2196/jmir.1559

Litvin, E. B., Abrantes, A. M., \& Brown, R. A. (2013). Computer and mobile technology-based interventions for substance use disorders: an organizing framework. Addictive Behaviors, 38(3), 1747-1756.

Malmberg, M., Kleinjan, M., Overbeek, G., Vermulst, A., Monshouwer, K., Lammers, J., ... Engels, R. C. (2014). Effectiveness of the 'Healthy School and Drugs' prevention programme on adolescents' substance use: a randomized clustered trial. Addiction, 109(6), 1031-1040. doi: 10.1111/add.12526

Newton, N. C., Teesson, M., Vogl, L. E., \& Andrews, G. (2010). Internet-based prevention for alcohol and cannabis use: final results of the Climate Schools course. Addiction, 105(4), 749-759. doi: 10.1111/j.1360-0443.2009.02853.x

Nordic Cochrane Centre Review Manager. (2014). RevMan (Computer program) Version 5.3. Copenhagen: The Cochrane Collaboration.

Oficina de las Naciones Unidas contra la Droga y el Delito (2013). Informe Mundial sobre las Drogas 2013. Recuperado de https:// www.unodc.org/doc/wdr2013/World_Drug_Report_2013_ Spanish.pdf

Oficina de las Naciones Unidas contra la Droga y el Delito. (2017). Informe Mundial sobre las Drogas 2017. Recuperado de https://www.unodc.org/doc/wdr2016/WDR_2016_ExSum_ spanish.pdf

Ondersma, S. J., Svikis, D., Thacker, L., Beatty, J. R., \& Lockhart, N. (2014). Computer-delivered screening and brief intervention (e-SBI) for postpartum drug use: a randomized trial. Journal of Substance Abuse Treatment, 46(1), 52-59. doi: 10.1016/j. jsat.2013.07.013

Palfai, T. P., Saitz, R., Winter, M., Brown, T. A., Kypri, K., Goodness, T. M., ... Lu, J. (2014). Web-based screening and brief intervention for student marijuana use in a university health center: pilot study to examine the implementation of eCHECKUP TO GO in different contexts. Addictive Behaviors, 39(9), 1346-1352. doi: 10.1016/j.addbeh.2014.04.025

Riper, H., Spek, V., Boon, B., Conijn, B., Kramer, J., Martin-Abello, K., ... Smit, F. (2011). Effectiveness of E-self-help interventions for curbing adult problem drinking: a meta-analysis. Journal of Medical Internet Research, 13(2), e42. doi:10.2196/jmir.1691

Rooke, S., Copeland, J., Norberg, M., Hine, D., \& McCambridge, J., (2013). Effectiveness of a self-guided web-based cannabis treatment program: randomized controlled trial. Journal of Medical Internet Research, 15(2), e26. doi: 10.2196/jmir.2256

Rooke, S., Webb, L., Albertella, L., \& Copeland, J., (2015). A smartphone application for managing cannabis use. Drug and Alcohol Dependence, 156, e192. doi: 10.1016/j.drugalcdep.2015.07.516 Schaub, M. P., Wenger, A., Berg, O., Beck, T., Stark, L., Buehler, E., ... Haug, S. (2015). A web-based self-help intervention with and without chat counseling to reduce cannabis use in problematic cannabis users: three-arm randomized controlled trial. Journal of Medical Internet Research, 17(10), e232. doi: 10.2196/jmir.4860

Schinke, S. P., Fang, L., \& Cole, K. C. A. (2011). Preventing substance abuse among black and hispanic adolescent girls: results from a computer-delivered, mother-daughter intervention approach. Substance Use \& Misuse, 46(1), 35-45. doi 10.3109/10826084.2011.521074

Schwinn, T. M., Schinke, S. P., \& Di Noia, J. (2010). preventing drug abuse among adolescent girls: outcome data from an internet-based intervention. Prevention Science: The Official Journal of the Society for Prevention Research, 11(1), 24. doi: 10.1007/ s11121-009-0146-9

Schwinn, T. M., Schinke, S. P., Hopkins, J., Keller, B., \& Liu, X (2017). An online drug abuse prevention program for adolescent girls: posttest and 1-year outcomes. Journal of Youth and Adolescence, 47(3), 490-500. doi:10.1007/s10964-017-0714-4

Shingleton, R. M., \& Palfai, T. P. (2016). Technology-delivered adaptations of motivational interviewing for health-related behaviors: a systematic review of the current research. Patient Education and Counseling, 99(1), 17-35. doi: 10.1016/j.pec.2015.08.005

Strecher, V. (2007). Internet methods for delivering behavioral and health-related interventions (eHealth). Annual Review of Clinical Psychology, 3, 53-76. doi: 10.1146/annurev. clinpsy.3.022806.091428

Substance Abuse and Mental Health Services Administration. (2012). Results from the 2011 National Survey on Drug Use and Health: summary of national findings (NSDUH Series H-44, HHS Publication No. 12-4713). Recuperado de https://www.samhsa.gov/ data/sites/default/files/Revised2k11NSDUHSummNatFindings/ Revised2k11NSDUHSummNatFindings/NSDUHresults2011.htm

Tait, R. J., \& Christensen, H. (2010). Internet-based interventions for young people with problematic substance use: a systematic review. Medical Journal of Australia, 192(11), S15.

Tait, R. J., Spijkerman, R., \& Riper, H. (2013). Internet and computer based interventions for cannabis use: a meta-analysis. Drug and Alcohol Dependence, 133(2), 295-304. doi: 10.1016/j.drugalcdep. 2013.05.012

Tait, R. J., McKetin, R., Kay-Lambkin, F., Carron-Arthur, B., Bennett, A., Bennett, K., ... Griffiths, K. M. (2015). Six-month outcomes of a web-based intervention for users of amphetamine-type stimulants: randomized controlled trial. Journal of Medical Internet Research, 17(4), e105.

Tiburcio, M., Lara, M. A., Aguilar Abrego, A., Fernández, M., Martínez Vélez, N., \& Sánchez, A. (2016). Web-based intervention to reduce substance abuse and depressive symptoms in Mexico: 
development and usability test. JMIR Mental Health, 3(3), e47. doi: 10.2196/mental.6001

Tossmann, D. H. P., Jonas, B., Tensil, M. D., Lang, P., \& Strüber, E. (2011). A controlled trial of an Internet-based intervention program for cannabis users. Cyberpsychology, Behavior and Social Networking, 14(11), 673-679. doi:10.1089/cyber.2010.0506.

Välimäki, M., Athanasopoulou, C., Lahti, M., \& Adams, C. E. (2016). Effectiveness of social media interventions for people with schizophrenia: a systematic review and meta-analysis. Journal of Medical Internet Research, 18(4), e92. doi: 10.2196/jmir.5385

Vogl, L. E., Newton, N. C., Champion, K. E., \& Teesson, M. (2014). A universal harm-minimisation approach to preventing psychostimulant and cannabis use in adolescents: a cluster randomised controlled trial. Substance Abuse Treatment, Prevention, and Policy, 9, 24. doi: 10.1186/1747-597X-9-24
Vona, P., Wilmoth, P., Jaycox, L. H., McMillen, J. S., Kataoka, S. H., Wong, M., ... Stein, B. D. (2014). A web-based platform to support an evidence-based mental health intervention: lessons from the CBITS web site. Psychiatric Services, 65(11), 13811384. doi:10.1176/appi.ps.201300512

Walton, M. A., Bohnert, K., Resko, S., Barry, K. T., Chermack, S. T., Zucker, R. A., ... Blow, F. C. (2013). Computer and therapist based brief interventions among cannabis-using adolescents presenting to primary care: one year outcomes. Drug and Alcohol Dependence, 132(3), 646-653. doi: 10.1016/j.drugalcdep.2013.04.020 Walton, M. A., Resko, S., Barry, K. L., Chermack, S. T., Zucker, R. A., Zimmerman, M. A.,... Blow, F. C. (2014). A randomized controlled trial testing the efficacy of a brief cannabis universal prevention program among adolescents in primary care. Addiction, 109(5), 786-797. doi:10.1111/add.12469 